\title{
Approximate Solutions for Mixed Boundary Value Problems by Finite-Difference Methods
}

\author{
By V. Thuraisamy*
}

\begin{abstract}
For mixed boundary value problems of Poisson and/or Laplace's equations in regions of the Euclidean space $E_{n}, n \geqq 2$, finite-difference analogues are formulated such that the matrix of the resulting system is of positive type. Discretization errors are established in a manner to reveal the continuous dependence of the rate of convergence on the smoothness of the solution. Isolated data singularities and their application to exterior problems are also discussed.
\end{abstract}

1. Introduction. In this paper we are concerned with approximating the solution $u(x)$ of the mixed boundary value problem

$$
\begin{aligned}
-\Delta u(x) & =f(x), & & x \in R, \\
\frac{\partial u(x)}{\partial n}+\alpha(x) u(x) & =g_{1}(x), & & x \in \partial R_{1}, \\
u(x) & =g_{2}(x), & & x \in \partial R_{2} .
\end{aligned}
$$

The region $R$ is a bounded connected set in the $n$-dimensional Euclidean space $E_{n}$, the boundary $\partial R$ of $R$ is $\partial R_{1} \cup \partial R_{2}$ and $\partial R_{1}=\partial R_{1}{ }^{(1)} \cup \partial R_{1}{ }^{(2)}$. In general each of $\partial R_{1}{ }^{(1)}, \partial R_{1}{ }^{(2)}$ and $\partial R_{2}$ may be a union of a finite number of surface elements. With $x \equiv\left(x_{1}, x_{2}, \cdots, x_{n}\right), \Delta \equiv \sum_{i=1}^{n} \partial^{2} / \partial x_{i}{ }^{2}, \partial / \partial n$ is the outward normal derivative and $f, g_{1}, g_{2}$ are given functions. $\alpha(x)$ is a piecewise differentiable function on $\partial R_{1}$. Existence, uniqueness and regularity of the solution of (1.1) is discussed, e.g., in [6], [11], [14], [17].

We restrict $\alpha(x)$ to be nonnegative and let it be zero on $\partial R_{1}{ }^{(1)}$ and positive on $\partial R_{1}{ }^{(2)}$. We also assume that $\partial R_{2} \neq \equiv \partial$ and that if $\partial R_{2}=\varnothing$ then there is a surface element of nonzero measure in $\partial R_{1}{ }^{(2)}$. We sometimes refer to $\partial R_{1}{ }^{(1)}$ as the 'Neumann piece' or the surface where the 'Neumann data' are prescribed, with similar nomenclature for the other boundary sets.

Finite-difference approximations to this problem have been studied by several authors (see e.g., [1], [2], [13], [18]) for the case $n=2$, where second-order convergence is established only in [2]. In [7], [12], second-order local approximations to the boundary operators are given without convergence proofs. We shall use the scheme in [2] for the plane and also develop others which are valid in all dimensions. All our analogues lead to matrices of positive type (see [3] for definitions).

In Sections 5, 6 and 7 we consider the question of reducing the regularity assumptions on the data for problem (1.1) when $f(x) \equiv 0$. We shall refer to this prob-

Received September 5, 1967, revised July 1, 1968.

* Except for minor improvements, the results of this paper were obtained as part of the author's Doctoral dissertation under the direction of Professor B. E. Hubbard at the Institute for Fluid Dynamics and Applied Mathematics and the research was supported in part by the U. S. Atomic Energy Commission under Contract AEC-AT-(40-1) 3443 and by the National Science Foundation under Grant NSF-GP-6631. 
lem as (1.1)'. The last three sections deal with isolated singularities and exterior problems. The analyses here are along the same lines as in [4] and [5] where the authors discuss the Dirichlet problem (see also [9], [10], [15], [16]).

2. Difference Approximations. Using uniform mesh-spacing of width $h$, we denote by $R_{h}$ the set of mesh-points in $R$ and by $\partial R_{h}$ the points common to the meshlines and $\partial R$. From now on a mesh-point shall always mean a member of $\bar{R}_{h}=R_{h} \cup \partial R_{h}$. We define $N(x) \subset \bar{R}_{h}$ to be the set of $2 n$ 'neighbors' of $x$ which are no further than a distance $h$ from $x$. If $N(x) \subset R_{h}$ we say that $x$ is in the set $R_{h}{ }^{\prime}$ of regular interior points and set $R_{h}{ }^{*}=R_{h}-R_{h}{ }^{\prime} . L(x)$ constitutes the closed line segments connecting $x$ to its $2 n$ neighbors. Using a multi-index $\alpha=\left(\alpha_{1}, \alpha_{2}, \cdots, \alpha_{n}\right)$, $|\alpha|=\alpha_{1}+\alpha_{2}+\cdots+\alpha_{n}$, we write any derivative of order $|\alpha|$ as $D^{\alpha} u$ and use the notation $M_{k}(u)$ (or just $M_{k}$ ) to indicate constants which depend on $\sup _{0 \leqq l \leqq k} D^{l} u$ over any specified set. Unspecified $K$ and $l$ shall always denote generic constants.

For the discrete Laplacian $\Delta_{h}$ in $R_{h}{ }^{\prime}$, we take the usual $(2 n+1)$ point operator. I.e.,

$$
\begin{aligned}
& U_{x_{i} \bar{x}_{i}}(x) \equiv h^{-2}\left\{U\left(x+h_{i}\right)-2 U(x)+U\left(x-h_{i}\right)\right\}, \\
& \Delta_{h} U(x)=\sum_{i=1}^{n} U_{x_{i} \bar{x}_{i}}(x),
\end{aligned}
$$

where the vector $h_{i}$ has $j$ th component $h \delta_{i j}, i=1, \cdots, n$. For $u(x) \in C^{4}(L(x))$

$$
\Delta_{h} u(x)-\Delta u(x)=\frac{h^{2}}{24} \sum_{i=1}^{n}\left\{\frac{\partial^{4} u\left(\xi^{(i)}\right)}{\left(\partial x_{i}\right)^{4}}+\frac{\partial^{4} u\left(\eta^{(i)}\right)}{\left(\partial x_{i}\right)^{4}}\right\}
$$

where $\xi^{(i)}, \eta^{(i)}$ are intermediate points on $L(x)$ satisfying $x_{i}-h<\xi_{i}{ }^{(i)}<x_{i}<$ $\eta_{i}{ }^{(i)}<x_{i}+h$ and $x_{j}=\xi_{j}{ }^{(i)}=\eta_{j}{ }^{(i)}$ for $j \neq i$. For $x \in R_{h}{ }^{*}$ let $x-\beta_{i} h_{i}, x+\alpha_{i} h_{i}$, $0<\alpha_{i}, \beta_{i} \leqq 1$, be the two neighboring mesh-points of $x$ in $\bar{R}_{h}$ lying on the meshline through $x$ in the $i$ th direction. We then define

$$
U_{x_{i} \bar{x}_{i}}^{(1)}(x) \equiv \frac{2}{\left(\alpha_{i}+\alpha_{i}\right) h^{2}}\left\{\frac{U\left(x+\alpha_{i} h_{i}\right)}{\alpha_{i}}-\left(\frac{1}{\alpha_{i}}+-\frac{1}{\beta_{i}}\right) U(x)+\frac{U\left(x-\beta_{i} h_{i}\right)}{\beta_{i}}\right\}
$$

Again if $u(x) \in C^{3}(L(x))$, then

$$
\Delta_{h}{ }^{(1)} u(x)-\Delta u(x)=\frac{h}{3} \sum_{i=1}^{n}\left\{\frac{\alpha_{i}{ }^{2}}{\left(\alpha_{i}+\beta_{i}\right)} \frac{\partial^{3} u\left(\eta^{(i)}\right)}{\left(\partial x_{i}\right)^{3}}-\frac{\beta_{i}{ }^{2}}{\left(\alpha_{i}+\beta_{i}\right)} \frac{\partial^{3} u\left(\xi^{(i)}\right)}{\left(\partial x_{i}\right)^{3}}\right\}
$$

where, now, $x_{i}-\beta_{i} h<\xi_{i}{ }^{(i)}<x_{i}<\eta_{i}{ }^{(i)}<x_{i}+\alpha_{i} h$ and $x_{j}=\xi_{j}{ }^{(i)}=\eta_{j}{ }^{(i)}$ for $j \neq i$. For $x \in R_{h}{ }^{*}$ we have a second approximation $\Delta_{h}{ }^{(0)}$ through

$$
U_{x_{i^{x_{i}}}}^{(0)}(x) \equiv \frac{\alpha_{i}+\beta_{i}}{2} U_{x_{i^{x}} \bar{x}_{i}}^{(1)}(x) .
$$

When (2.5) is used, the 'reduced' matrix obtained by deleting rows and columns corresponding to boundary points is symmetric, but since the approximation is only of order zero its usefulness is limited.

We shall now develop approximations for the boundary operator. In two dimensions a simple first-order approximation to the normal derivative can be constructed by choosing two points in $\mathrm{R}_{h}$, one on each side of the normal, together with 
the boundary point, such that, the resulting matrix is of positive type. To generalize to higher dimensions: at a boundary point $x$ we choose a local cartesian coordinate system such that the $n$th coordinate direction coincides with the interior normal.
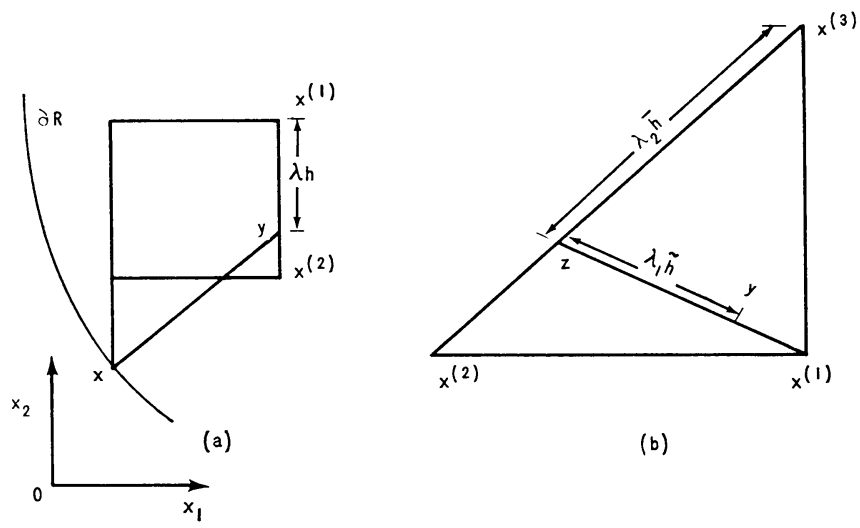

(b)

Figure 1

For any point $x^{(j)} \in R$ such that the line segment $x x^{(j)}$ is in $\bar{R}$ we have, if $u \in C^{2}(\bar{R})$,

$$
u\left(x^{(j)}\right)=u(x)+\sum_{i=1}^{n} x_{i}{ }^{(j)} u_{x_{i}}(x)+M_{2} h^{2} .
$$

We pick $n$ such points $x^{(j)}$ in $R_{h}$ (within $O(h)$ of $x$ ) and $n$ numbers $b_{j}$ such that

$$
(X)_{n \times n}(B)_{n \times 1}=\left[\begin{array}{c}
0 \\
0 \\
\cdot \\
\cdot \\
\cdot \\
0 \\
1
\end{array}\right]
$$

where the columns of the matrix $X$ are the coordinates of the point $x^{(j)}$ and we have written $B$ for the vector with components $b_{j}$. (2.6) implies that $\left|b_{j}\right|<K h^{-1}, j=1$, $\cdots, n . X$ is nonsingular as soon as the points $x^{(j)}$ are not in an $(n-2)$-dimensional manifold and $B>0$ (required for positivity of the matrix) when all the cofactors of elements of the last row are of the same sign. Another first-order approximation follows. First for $n=2$, if $u \in C^{2}(\bar{R})$, then for points as in Fig. 1(a) we may write

$$
\frac{u(x)-(1-\lambda) u\left(x^{(1)}\right)-\lambda u\left(x^{(2)}\right)}{|x-y|}=u_{n}(x)+M_{2} h .
$$

When $n=3, y$ can be chosen in the closure of a right triangle with two sides equal to $h$ as in Fig. 1(b), where $x^{(1)}, x^{(2)}, x^{(3)}$ are three of the vectices of a meshsquare. Similarly for $n \geqq 4$ we take $y$ in an $n$-hedron with $(n-1)$ edges equal to $h$ such that $|x-y|$ is uniformly less than $l h$ (where we can take $l \leqq(n+3)^{1 / 2}$ if $2 h<$ infimum of the radius of curvature of $\partial R$ ) and we have in general 


$$
\frac{u(x)-\sum_{i=1}^{n} \nu_{i} u\left(x^{(i)}\right)}{|x-y|}=\frac{\partial u}{\partial n}(x)+O(h)
$$

where the $\nu_{i}$ are nonnegative and their sum is unity. Thus we can define a first-order approximation to the normal derivative either using (2.7) or using (2.9) by

$$
\delta_{1} u(x) \equiv \sum_{i=1}^{n} b_{i}\left\{u(x)-u\left(x^{(i)}\right)\right\}
$$

such that $0<h b_{i} \leqq K$ for $i=1,2, \cdots, n$.

3. Discrete Analogues. Based on the several difference operators developed in the last section, we shall now write discrete analogues of (1.1), following the definition of an operator $B_{1}$ introduced for notational convenience.

$$
\begin{array}{rlrl}
B_{1} U(x) & \equiv \delta_{1} U(x)+\alpha(x) U(x), \\
-\Delta_{h} u(x, h) & =f(x), & x \in R_{h}, \\
B_{1} u(x, h) & =g_{1}(x), & x \in \partial R_{1, h}, \\
u(x, h) & =g_{2}(x), & x \in \partial R_{2, h} .
\end{array}
$$

We note that Eqs. (3.2) actually represent four distinct sets of equations depending on the choice of operators in $R_{h}{ }^{*}$ and on $\partial R_{1, h}$. The following lemma is an easy consequence of the foregoing definitions.

Lemma 1 (Maximum Principle). If a mesh-function is such that

$$
\begin{aligned}
-\Delta_{h} V(x) \geqq 0, & x \in R_{h}, \\
B_{1} V(x) \geqq 0, & x \in \partial R_{1, h}, \\
V(x) \geqq 0, & x \in \partial R_{2, h},
\end{aligned}
$$

tlen

$$
V(x) \geqq 0 \text { for all } x \in \bar{R}_{h} \text {. }
$$

We now define discrete analogues of Green's functions to systems (3.2). We shall call these Robin's functions. For $y \in \bar{R}_{h}$, let $R(x, y, h)$ be such that

$$
\begin{aligned}
-\Delta_{h, x} R(x, y, h) & =h^{-n} \delta(x, y), \quad x \in R_{h}, \\
B_{1, x} R(x, y, h) & =h^{-n+1} \delta(x, y), \quad x \in \partial R_{1, h}, \\
R(x, y, h) & =\delta(x, y), \quad x \in \partial R_{2, h},
\end{aligned}
$$

where

$$
\begin{aligned}
\delta(x, y) & =1, & & x=!, \\
& =0, & & x \neq y .
\end{aligned}
$$

The suffixes $x$ on the operators $\Delta_{h}$ and $B_{1}$ indicate that the operation is with respect to $x$, holding $y$ as a parameter.

From (3.2) and (3.4) follow the important property that

$$
R(x, y, h) \geqq 0 \quad \text { for all } x, y \in \bar{R}_{h} .
$$


Lemma 2 (Representation Theorem). For any mesh-function $V(x)$ we have

$$
\begin{aligned}
V(x) \equiv h^{n} & \sum_{y \in R h} R(x, y, h)\left[-\Delta_{h} V(y)\right]+h^{n-1} \sum_{y \in \partial R_{1, h}} R(x, y, h) B_{1} V(y) \\
& +\sum_{y \in \partial R_{2, h}} R(x, y, h) V(y) .
\end{aligned}
$$

4. Some Basic Bounds. To establish convergence of a discrete solution $u(x, h)$ of (3.2) to the continuous one of (1.1) we need to establish bounds on various discrete sums of Robin's functions. These are collected in

Lemma 3. If (i) $\alpha(x) \geqq \alpha_{0}>0$ for $x \in \partial R_{1}$ or (ii) $\partial R \in C^{2, \lambda}, \lambda>0$ and $\Delta_{h}{ }^{(1)}$ is used over $R_{h}{ }^{*}$, then each Robin's function satisfies the inequalities

$$
\begin{aligned}
h^{n-1} \sum_{y \in R_{h}} R(x, y, h) \gamma(y) & \leqq K, \\
h^{n-1} \sum_{y \in \partial R_{1}} R(x, y, h) & \leqq K, \\
\sum_{y \in \partial R_{2, h}} R(x, y, h) & \leqq K, \\
h^{n} \sum_{y \in R_{h}} R(x, y, h) & \leqq K .
\end{aligned}
$$

The quantity $\gamma(y)$ is a function of the $\alpha_{i}, \beta_{i}$ only (see (2.3), (2.5)).

Proof. (i) The proofs are accomplished by introducing special grid functions. E.g., taking $\Delta_{h}{ }^{(1)}$ over $R_{h}{ }^{*}$, if we define $w(x)$ to be a constant $\lambda_{0}$ over $R_{h}$ and $\lambda_{0}-\tau h$ over $\partial R_{h}, \tau>0$, then substitution of this $w(x)$ into (3.6) yields the first three inequalities. For the last one we pick the 'square of the distance' function as $w(x)$.

(ii) If condition (i) of the lemma is not met, then we have to assume conditions (ii). With this assumption we simply use the fact [2] that there is a function $\phi(x) \in C^{2, \lambda}(\bar{R})$ such that

$$
\begin{aligned}
-\Delta \phi(x) \geqq 2, & x \in R, \\
\partial \phi(x) / \partial n+\alpha(x) \phi(x) \geqq 2, & x \in \partial R_{1} .
\end{aligned}
$$

Utilizing Lemma 3 and the representation formula (3.6) it is now a simple matter to establish the following convergence theorem.

Theorem 1. Let $u \in C^{3}(\bar{R})$ be the solution of (1.1). Then, with $e(x, h)=u(x)$ - $u(x, h)$, we have uniformly in the maximum norm

$$
|e(x, h)|_{\bar{R}_{h}} \leqq K h .
$$

Observing that in most practical cases we can construct discrete analogues to (1.1) as well as smooth functions satisfying (4.2) even when the boundary is only piecewise smooth, we look upon case (ii) of the lemma more as a theoretical restriction. Thus if certain smoothness of the unknown function $u(x)$ in (1.1) is known a priori, we can often give some convergence results.

5. Reduced Regularity Assumptions. In this and the following two sections we consider the weakening of the regularity assumptions for problem $(1.1)^{\prime}$ (i.e., when $f(x) \equiv 0)$. We begin with the statement of some results established in [4].

Lemma 4. If $u \in C^{m, \lambda}(\bar{R})$ and $\Delta u=0$ in $R$, then the local errors, i.e., the difference 
between the continuous and discrete Laplacians, satisfy the inequalities

$$
\begin{aligned}
\left|\Delta_{h}{ }^{(i)} u(x)\right| & \leqq K\left[h^{m+\lambda-2}+h^{i}\right], & & i=0,1, \quad x \in R_{h}{ }^{*} . \\
\left|\Delta_{h} u(x)\right| & \leqq K\left[h^{m+\lambda-2}+h^{2}\right], & & x \in R_{h}{ }^{\prime} .
\end{aligned}
$$

If, in addition, $d(x) \geqq 2 h$, then we also have

$$
\begin{aligned}
\left|\Delta_{h} u(x)\right| & \leqq K h^{2} d(x)^{\lambda+m-4}, \quad m=1,2,3, \\
& \leqq K h^{2}, \quad m \geqq 4 .
\end{aligned}
$$

In the above statement $d(x)=\min _{y} \in \partial R|x-y|$ and we recall that the superfix $i$ of (5.1) selects the appropriate discrete Laplacian. It is important to note that, except when $m=1, i=1$, the $K$ in (5.1) is independent of the $\alpha_{i}, \beta_{i}$. In the exceptional case it can be shown that the $K$ is of the form

$$
\bar{K}\left(\sum_{i=1}^{n} \frac{1}{\alpha_{i}+\beta_{i}}\right)
$$

where $\bar{K}$ is now independent of $\alpha_{i}, \beta_{i}$ as well as $h$. Since (5.4) may be unbounded as $h \rightarrow 0$, we require the following lemma, which may be proved by using a meshfunction which is unity in $R_{h}$ and vanishes on $\partial R_{h}$.

Lemma 5. Writing $R^{(1)}(x, y, h)$ for the Robin's function corresponding to ${\Delta_{h}}^{(1)}$ over $R_{h}{ }^{*}$, we have

$$
h^{n} \sum_{y \in R_{h}{ }^{*}} R^{(1)}(x, y, h) \zeta^{(1)}(y) \leqq K h,
$$

where $\zeta^{(1)}(y)$ depends only on the $\alpha_{i}$ and $\beta_{i}$.

On applying Lemma 4, e.g., to the case $m=1$, one finds that the error can be uniformly limited to $O\left(h^{\lambda}\right)$ if the basic bounds are sufficiently improved over the set

$$
R_{h, \delta}^{\prime} \equiv\left\{x \in R_{h}{ }^{\prime} \mid d(x) \leqq \delta\right\}
$$

for a $\delta>0$ independent of $h$. It turns out that we can easily extend an estimate of type (5.5) (without the factor $\zeta^{(1)}$ ) to a boundary strip of width $O(h)$ (Lemma 6), but for the rest of $R_{h, \delta}^{\prime}$ rather complex analysis appears unavoidable. This we do in the next section. by

Definition 1. For $h$ sufficiently small let $R_{0}{ }^{*}=R_{h}{ }^{*}$ and define $R_{l}{ }^{*}, l=1,2, \cdots$,

$$
R_{l}{ }^{*}=\left\{x \in R_{h}{ }^{\prime} \mid N(x) \cap R_{l-1} \neq \varnothing, x \in R^{*}(l-1) \bigcup_{j=0}^{l-1} R_{j}^{*}\right\} .
$$

We note here that, when $h$ is small enough, for every $x \in R_{0}{ }^{*}$ there is $y \in N(x)$ such that $y$ is also in $R_{1}{ }^{*}$. This would then imply that mesh-points in $R_{h}-R^{*}(l+1)$ would be at least a distance $l h$ from $\partial R$.

Lemma 6. For l a positive integer,

$$
h^{n} \sum_{y \in R^{*}(l)} R(x, y, h) \leqq K h .
$$

Proof. For $k=1,2, \cdots, l$, define $w_{k}(x)$ as zero on $\partial R_{h} \cup R^{*}(k)$ and unity otherwise. 
6. A Basic Lemma. As suggested by the discussion following Lemma 5, we shall, in this section, develop a basic lemma (Lemma 7) that is crucial in the proof of the convergence theorem (Theorem 2 ) under reduced regularity conditions. The idea is to include with $R(x, y, h)$ as singular a factor $d(y)^{-\rho}(\rho>0)$ as possible and attempt to obtain bounds of the type (4.1d). We begin with the definition of yet another subset of $R_{h}$.

Definition 2 . For a fixed positive number $\delta$, let

$$
T(l, \delta)=\left\{x \in R_{h} \mid x \in R^{*}(l), d(x)<\delta\right\} .
$$

Lemma 7. For $l$ and $\delta$ independent of $h$ and $\delta$ constrained as required in Lemma 8 , if $\partial R \in C^{2}$, then

$$
h^{n} \sum_{y \in T(l, \delta)} R(x, y, h) d(y)^{-1-\epsilon} \leqq K(\epsilon) h^{-\epsilon}
$$

where $\epsilon$ can be made smaller than any given small positive number but $K(\epsilon)$ may be unbounded as $\epsilon \rightarrow 0$.

Before we can prove this lemma we need some preliminary results. We take an arbitrary point $x \in T(l, \delta)$. The normal to $\partial R$ at $t$ through the mesh-point $x$ makes an angle $\theta_{i}$ with the $x_{i}$ direction. Denoting by $\gamma_{t}$ the $(n-1)$-dimensional tangent plane at $t$, we let $y$ be the foot of the normal to the plane from $x+h_{i} \equiv e . e p$ is the normal to $\partial R$ through $e$ and meeting $\gamma_{t}$ at $r$. Also $p q$ is normal to this plane and the angle between $e y$ and $e p$ is denoted by $\phi_{i}$.

Definition 3. For $x \in \partial R$ let $r_{i}(x), r_{e}(x)$ be the radii of the largest open balls in $R$ and $\sim R$ (complement of $R$ ) respectively, such that they are also tangent to $\partial R$ at $x$, and let $r_{0}$ be given by

$$
r_{0}=\min _{x \in \partial R}\left\{r_{i}(x), r_{e}(x)\right\} .
$$

Lemma 8 . With $\delta \leqq r_{0} / 3$ and $\partial R \in C^{2}$, we have

$$
\begin{aligned}
\left|\phi_{i}\right| & \leqq K h, \\
\left|d\left(\left(x+h_{i}\right)-|e-y|\right)\right| & \leqq K h^{2} .
\end{aligned}
$$

The proof of this lemma may be found in [4]. We shall use Lemma 8 to prove the following

Lemma 9. Let $N_{0}$ be a positive integer and $\epsilon$ be given by

$$
\epsilon^{-1}=2^{N_{0}}+1 \text {. }
$$

Then

$$
|| e-\left.y\right|^{1-\epsilon}-d\left(x+h_{i}\right)^{1-\epsilon} \mid \leqq K h^{2} d(x)^{-\epsilon} .
$$

Proof. For any two positive numbers $a, b$ and a positive integer $N$ we have

$$
a-b=\left(a^{1 / N}-b^{1 / N}\right)\left(a^{1-1 / N}+a^{1-2 / N} b^{2 / N}+\cdots+b^{(N-1) / N}\right) .
$$

Since $2^{N_{0}}+1$ is an integer, we can let $N=1 / \epsilon$ and write

$$
\left|a^{\epsilon}-b^{\epsilon}\right| \leqq|a-b|\left\{a^{1-\epsilon}+a^{1-2 \epsilon} b^{\epsilon}+\cdots+b^{1-\epsilon}\right\}^{-1}
$$

where the second factor on the right is a finite series of $\left(2^{N_{0}}+1\right)$ terms. 
We employ (6.8) with $a=|e-y|, b=d\left(x+h_{i}\right)$ and the fact that, for $l$ in $T(l, \delta)$ large enough,

$$
d(x) \leqq K \min \left\{d\left(x+h_{i}\right),|e-y|\right\}
$$

to obtain from (6.4) the inequality

$$
|| e-\left.y\right|^{\epsilon}-d\left(x+h_{i}\right)^{\epsilon} \mid \leqq K h^{2} d(x)^{\epsilon-1} .
$$

Now, for any real $\epsilon, a, b$ and nonnegative integers $N$

$$
a^{1-2^{N_{\epsilon}}}-b^{1-2^{N_{\epsilon}}}=\frac{(a+b)+(a b)^{2^{N_{\epsilon}}}\left\{a^{1-2^{N+1} \epsilon}-b^{1-2^{N+1} \epsilon}\right\}}{a^{2 N_{\epsilon}}+b^{2^{N_{\epsilon}}}} .
$$

Consequently we may write $\left\{a^{1-\epsilon}-b^{1-\epsilon}\right\}$ as a continued fraction of the form

$$
a^{1-\epsilon}-b^{1-\epsilon}=\frac{a-b+(a b)^{\epsilon}\left[\frac{a-b+(a b)^{2 \epsilon}\left\{\frac{a-b+(a b)^{4 \epsilon}\left(\frac{\cdots}{\cdots}\right)}{a^{4 \epsilon}+b^{4 \epsilon}}\right\}}{a^{2 \epsilon}+b^{2 \epsilon}}\right]}{a^{\epsilon}+b^{\epsilon}}
$$

But since $\epsilon$ satisfies (6.5) we may write (6.12) in the form

$$
\begin{aligned}
a^{1-\epsilon}-b^{1-\epsilon}=\frac{1}{a^{\epsilon}+b^{\epsilon}}[(a-b)\{1 & \left.+\sum_{N=0}^{N_{0-2}} \frac{(a b)^{\epsilon} \sum_{M=0}^{N} 2^{M}}{\prod_{L=0}^{N}\left(a^{2 L^{L+1}}+b^{2 L_{1 \epsilon}}\right)}\right\} \\
& \left.+\left(a^{\epsilon}-b^{\epsilon}\right)\left\{\frac{(a b)^{\epsilon}\left(\sum_{M=0}^{N_{0-1}} 2^{M}\right)}{\prod_{L=1}^{N_{0}-1}\left(a^{2^{L_{\epsilon}}+b^{L_{\epsilon}}}\right)}\right\}\right] .
\end{aligned}
$$

Now we let $a=|e-y|, b=d\left(x+h_{i}\right)$ in (6.13), employ (6.9), (6.10), (6.4) and the additional facts that $d\left(x+h_{i}\right)$ and $|e-y|$ are bounded by $K d(x)$ to complete the proof.

We remark here that the choice of the point $x+h_{i}$, as $e$ was only for convenience of notation. The proof applies for any point within $O(h)$ of $x$ and in $T(l, \delta)$.

Proof of the Lemma. Given the results of Lemma 9, the proof of Lemma 7 is now routine. As in [4] it can be shown that for $0<\mu<1$ and appropriate $\delta$, the negative of the discrete Laplacian of $d(x)^{\mu}$ exceeds $\lambda d(x)^{\mu-2}$ for a positive $\lambda$. Then by defining a $C^{\infty}$ function which equals $1 / \lambda$ over a 'boundary' strip of width $r_{0} / 3$ and substituting it into the representation formula we obtain the lemma.

7. Error Estimates. In this section we state and comment on the main results on the continuously improving rates of convergence of the various approximate solutions to $(1.1)^{\prime}$, with increasing regularity assumptions. In [2] the authors developed a 4-point boundary operator with second-order accuracy for the twodimensional case, but with the assumption that for $x \in \partial R_{1}{ }^{(2)}, \alpha(x)$ exceeds some $\alpha_{0}>0$. The results of the last two sections hold also when this operator $\delta_{2}$ (say) is substituted for $\delta_{1}$ in (3.1). Hence we shall state the theorem separately for the cases $n=2$ and $n>2$. In (7.1), $\delta_{2}$ must be used for convergence higher than $O(h)$.

Theorem 2. Let $u(x)$ be the solution to problem $(1.1)^{\prime}$ and $u(x, h)$ to one of the discrete analogues in (3.2). If $\partial R \in C^{2}, u(x) \in C^{m, \lambda}(\bar{R})$ and $e(x, h) \equiv u(x)-u(x, h)$, then there is an $h_{0}$ such that for all $h \leqq h_{0}$, we have

(i) when $n=2, i=0,1\left(\right.$ from $\left.\Delta_{h}{ }^{(i)}\right)$ 


$$
\begin{aligned}
|e(x, h)|_{\bar{R}_{h}} & \leqq K h^{\lambda}, \quad m=1, \\
& \leqq K h\left[h^{\lambda}+h^{i}+h^{1-\epsilon}\right], \quad m=2, \\
& \leqq K\left[h^{2-\epsilon}+h^{i+1}\right], \quad m=3, \\
& \leqq K h^{i+1}, \quad m \geqq 4 ;
\end{aligned}
$$

(ii) when $n \geqq 3$

$$
\begin{aligned}
|e(x, h)|_{R_{h}} & \leqq K h^{\lambda}, \quad m=1, \\
& \leqq K h, \quad m \geqq 2 .
\end{aligned}
$$

The reader will have no difficulty supplying the proof. Lemmas 4, 6 and 7 together with inequalities of the type

$$
\begin{aligned}
d(y)^{-2} & \leqq K d(y)^{-1-\epsilon}, \quad \text { if } \lambda+\epsilon-1 \geqq 0, \\
& \leqq K d(y)^{-1-\epsilon} h^{\lambda+\epsilon-1}, \quad \text { if } \lambda+\epsilon-1<0,
\end{aligned}
$$

are used with $e(x, h)$ for $V(x)$ in (3.6) ((7.3) is for case (i), $m=2)$. It is important to observe that when summing over $R_{h}{ }^{*}$, in all cases except when $m=1, i=1$, the constants $K$ are also independent of $\alpha_{i}$ and $\beta_{i}$. In the exceptional case (Lemma 4), however, we can use Lemma 5 so that in all cases of Theorem 2 the constant $K$ remains bounded as $h \rightarrow 0$ for every fixed $\epsilon$ in the appropriate set.

8. Isolated Singularities. In Sections 8 and 9 we extend to the mixed boundary problem some of the results obtained for the Dirichlet problem in a recent paper [5] by Bramble, Hubbard and Zlamal. $u(x)$ of (1.1) now allows isolated singularities. Specifically, we assume that

$$
\begin{aligned}
& u \in C^{M .0}(\bar{R}-\sigma), \\
&\left|D^{l} u(x)\right| \leqq K, \quad l \leqq m, \\
& \leqq K|x-\sigma|^{m+\lambda-l}, \quad m+1 \leqq l \leqq M,
\end{aligned}
$$

where $\sigma$ is an arbitrarily fixed point in $\bar{R}, m$ is an integer not exceeding 3 and $0<\lambda \leqq 1$. We now assume that the mesh is placed so that

$$
\min _{x \in \bar{R} ; x \text { on a mesh line }}|x-\sigma| / h=a>0 .
$$

Our success in proving convergence theorems will now depend on how closely we can majorize the Robin's functions. Let

$$
\begin{aligned}
\rho_{n}{ }^{2} & =|x-y|^{2}+\gamma_{n} h^{2}, \quad n \geqq 2, \\
v_{2}(|x-y|) & =\frac{1}{\Gamma_{2}} \log \left(D / \rho_{2}{ }^{2}\right), \quad n=2, \\
v_{n}(|x-y|) & =\rho_{n}{ }^{2-n} / \Gamma_{n}, \quad n \geqq 3, \\
\psi_{n}(x, y) & =v_{n}(|x-y|), \quad x \in R_{h} \cup \partial R_{2, h}, \quad n \geqq 2 . \\
& =v_{n}(|x-y|)+\delta_{n}(y), \quad x \in \partial R_{1, h}, \quad
\end{aligned}
$$


The functions $v_{n}$ resembling the continuous Green's functions have in fact been proved in [5] to be majorants for the discrete Green's functions when the quantities $\gamma_{n}, \Gamma_{n}$ (which depend only on $n$ ) are appropriately chosen. The expressions for $\gamma_{n}, \Gamma_{n}$ are too long to be repeated here. Our contribution, therefore, is in modifying the $v_{n}$ on the boundary such that the Laplacians and boundary operators operating on $\left[\psi_{n}(x, y)-R(x, y, h)\right]$ have the appropriate signs for $\psi_{n}$ to qualify as majorants for our Robin's functions. For this purpose we find that it is sufficient to take

$$
\delta_{n}(y)=K h /[d(y)]_{\partial R_{1}}
$$

where $[d(y)]_{\partial R_{1}}$ is the distance of $y$ from $\partial R_{1}$ and $K$ sufficiently large. We now state formally the

Lemma 10 . For $n \geqq 2$, let $y \in R_{h}-R^{*}\left(l_{n}\right)$ where $l_{n} \geqq \sqrt{ } n$ (except that $l_{2}$ may have to exceed $10 \mathrm{~h}$ when $\delta_{2}$ is the boundary operator). Then, taking note of the foregoing definition and observations, we have

$$
R(x, y, h) \leqq \psi_{n}(x, y)+K / d(y)^{n-1} .
$$

The weakness of this bound as $d(y) \rightarrow O(h)$ is reflected in the results of the convergence theorem which follows.

Theorem 3. Let $u(x)$ be the solution of (1.1) satisfying $(8.1)$ and $u(x, h)$ the appropriate solution of (3.2). If $d(\sigma)=d_{0}>0$, then the error in maximum norm $|e(x, h)|_{\bar{R}_{h}} \equiv|u(x)-u(x, h)|_{\bar{R}_{h}}$ is such that

(i) corresponding to $M=4, n=2$ and $\delta_{2}$,

$$
\begin{aligned}
|e(x, h)|_{\bar{R}_{h}} & \leqq K\left(\epsilon, d_{0}\right) h^{m+\lambda-\epsilon}, \quad 0<m+\lambda \leqq 2, \\
& \leqq K\left(\epsilon, d_{0}\right) h^{2}, \quad m+\lambda>2,
\end{aligned}
$$

(ii) and corresponding to $M=3, n \geqq 2$ and $\delta_{1}$,

$$
\begin{aligned}
|e(x, h)|_{\bar{R}_{h}} & \leqq K\left(\epsilon, d_{0}\right)\left\{h^{m+\lambda+n-2-\epsilon}|x-\sigma|^{2-n+\epsilon}+h\right\}, 2-n<m+\lambda \leqq 1, \\
& \leqq K\left(\epsilon, d_{0}\right) h, \quad m+\lambda>1,
\end{aligned}
$$

where $K\left(\epsilon, d_{0}\right)$ may be unbounded as $\epsilon \rightarrow 0$.

Proof. We shall briefly look at case (ii) for $n>2$. Let $\Omega$ be a closed ball with center $\sigma$ and radius $r$ such that $\Omega_{h} \equiv\left\{x \in R_{h} \cap \Omega\right\} \subset R_{h}{ }^{\prime}$. Then from Lemma 10 we have

$$
\begin{aligned}
|e(x, h)| \leqq & h^{n} \sum_{y \in \Omega_{h}}\left[\psi(x, y)+K d(y)^{-n+1}\right]|y-\sigma|^{\epsilon-n}|y-\sigma|^{m+\lambda-3+n-\epsilon} \cdot h \\
& +O(h) .
\end{aligned}
$$

If $y \in \Omega_{h},-n<p, q<0$, and $x, z$ are such that $|x-y| \geqq a h>0,|y-z| \geqq$ $a h>0$, then (see e.g., [5])

$$
h^{n} \sum_{y \in \Omega_{h}}|x-y|^{p}|y-z|^{q} \leqq K\left\{|x-z|^{n+p+q}+1\right\} .
$$

Taking $z=\sigma, p=2-n, q=\epsilon-n$, we have, for $x \neq y$,

$$
h^{n} \sum_{y \in \Omega_{n}} v_{n}(|x-y|)|y-\sigma|^{\epsilon-n} \leqq K|x-\sigma|^{2-n+\epsilon} .
$$


The term from the sum in (8.11) for $y=x$ is bounded by

$$
K h^{3}|x-\sigma|^{m+\lambda-3} \text {. }
$$

The last two inequalities together with the integral inequality

$$
\int|y-\sigma|^{\epsilon-n} d y \leqq K(\epsilon)
$$

used in (8.11) completes the proof.

We remark here that, using inequality (6.2), it can be verified that the dependence of $K\left(\epsilon, d_{0}\right)$ on $d_{0}$ is no worse than $O\left(d_{0}^{-1}\right)$ as $d_{0} \rightarrow O(h)$.

9. Exterior Problems. We shall consider only the case $n=2$. Let $R$ be a region that contains the point at infinity and $\tilde{D}$ be a region such that $R \cap \tilde{D}=\varnothing$. We place the origin $\sigma$ in $\widetilde{D}$ and map $R$ onto a region $D$ and $\partial R$ onto $\partial D$ by inversion about the unit circle with center $\sigma . \bar{D}$ is then a bounded region in $E_{2}$. We shall again consider the problem (1.1) which now becomes an exterior problem with unbounded $R$. For clarity we refer to this as (1.1)". Our method of solution is to write down an equivalent problem for (1.1)" over $\bar{D}$, solve it by the methods of this paper and deduce the approximate solution to (1.1)" from this. In this way we avoid having to deal with arbitrarily large, albeit finite, regions and nonuniform spacing.

Using polar coordinates $(r, \theta)$ for a point in $R$ we let

$$
v(\rho, \theta)=u(r, \theta), \quad \rho=1 / r .
$$

We denote the normal at $(\rho, \theta)$ to $\partial D$ by $\tilde{n}$. It can be shown by a somewhat laborious but elementary calculation that

$$
\frac{\partial v}{\partial \tilde{n}}(\rho, \theta)=r^{2} \frac{\partial u}{\partial n}(r, \theta), \quad(r, \theta) \in \partial R
$$

Also we have

$$
\Delta v(\rho, \theta)=\rho^{4} \Delta u(r, \theta), \quad(\rho, \theta) \in D .
$$

Hence equivalent to (1.1)" we have, with obvious notation,

$$
\begin{aligned}
-\Delta v(\rho, \theta) & =\rho^{-4} \tilde{f}(\rho, \theta), & (\rho, \theta) & \in D, \\
\frac{\partial v}{\partial \tilde{n}}(\rho, \theta)+\frac{\tilde{\alpha}(\rho, \theta)}{\rho^{2}} v(\rho, \theta) & =\frac{\tilde{g}_{1}(\rho, \theta)}{\rho^{2}}, & (\rho, \theta) & \in \partial D_{1}, \\
v(\rho, \theta) & =\tilde{g}_{2}(\rho, \theta), & (\rho, \theta) & \in \partial D_{2} .
\end{aligned}
$$

Since $\sigma$ can be chosen such that $[d \sigma]_{\partial D}$ exceeds a positive constant $\rho_{0},(9.4)$ is well defined with an interior singularity at $\rho=0$.

We assume that $u$ in (1.1)" is such that

$$
\begin{aligned}
u & \in C^{M}(\bar{R}-\infty) \\
\left|D^{l} u(x)\right| \leqq K|x|^{-n-l} & \text { as }|x| \rightarrow \infty, \quad 0 \leqq l \leqq M+1 .
\end{aligned}
$$

Let $v(x, h)$ be the solution of a discrete problem corresponding to (9.4) and let (8.2) hold (but now in $D$ ). Define 


$$
\begin{aligned}
& v(x, h)=u(x, h), \\
& e(x, h)=v(x, h)-v(x) .
\end{aligned}
$$

Then we also have

$$
e(\tilde{x}, h)=u(x, h)-u(x)=e(x, h) .
$$

Observing in addition that conditions (9.5) on $u$ imply

$$
\begin{gathered}
v \in C^{M}(\bar{D}-\sigma), \\
\left|D^{l} v(x)\right| \leqq K|x-\sigma|^{\eta-l} \quad \text { as } x \rightarrow \sigma
\end{gathered}
$$

we immediately have the following convergence results as a consequence of Theorem 3.

Theorem 4. If $u(x)$ satisfies (1.1)" and (3.3), then the discretization error $e(x, h)$ is such that, for $j=1$ or 2 in $\delta_{j}$ and $M=j+2$,

$$
\begin{aligned}
|e(x, h)|_{\bar{R}_{h}} & \leqq K(\epsilon) h^{\eta-\epsilon}, \quad 0<\eta \leqq j, \\
& \leqq K(\epsilon) h^{j}, \quad n>j .
\end{aligned}
$$

$\bar{R}_{h}$, of course, is now the set of points in $\bar{R}$ whose inverses are in $\bar{D}_{h}$.

We shall now look at a special case of (1.1) when $\sigma \in \partial R$. Theorem 3 is of course not valid when $\sigma \in \partial R$. Let $\partial R$ be convex at $\sigma$, and let there be an arc $\sigma z$ of $\partial R$ which is in $\partial R_{2}$. Let $f(x)$ in (1.1) be identically zero and take $\delta_{1}$ for the approximation of the normal derivative together with $\Delta_{h}{ }^{(1)}$ over $R_{h}{ }^{*}$. Let us also assume that in the construction of $\delta_{1}$ for $x \in \partial R_{1, h}$ there is always a connection in $R_{h}{ }^{\prime}$.

THEOREM 5. When the above statements hold, together with (8.2), we have

$$
|e(x, h)|_{\bar{s}_{h}} \leqq K h^{\nu}, \quad \nu \leqq m+\lambda, \nu<1 / 2,
$$

where $\bar{S}_{h}$ is the result of deleting points of $\bar{R}_{h}$ in a small $O(h)$ neighborhood of $\sigma$.

This theorem shall not be proved here as it can be easily adapted from the proof of a similar theorem of Wigley [18]. As an application of Theorem 5 we consider, e.g., the half-plane problem,

$$
\begin{aligned}
\Delta u\left(x_{1}, x_{2}\right) & =0, \quad x_{2}>0, \\
u(x) & =g_{2}(x), \quad x_{1} \rightarrow-\infty, x_{2}=0,
\end{aligned}
$$

with either type of boundary condition permitted on the rest of the $x_{1}$-axis. If $\sigma$ is the point $(0,-1)$ and $C$ is the unit circle $D$ with center $\left(0,-\frac{1}{2}\right)$ and radius $1 / 2$, then by solving an equivalent problem in $D$ as in Theorem 2 we approximate $u(x)$ of (9.9) with the error bounds given by

Theorem 6. If

$$
\begin{array}{rlrl}
\left|D^{l} u\right| & \leqq K|x|^{-\eta-l}, & & |x| \rightarrow \infty, \\
\left|g_{1}\right| \leqq K|x|^{-\eta-1}, & & |x| \rightarrow \infty, x_{2}=0,
\end{array}
$$

when appropriate and

$$
\left|g_{2}\right| \leqq K|x|^{-\eta}, \quad|x| \rightarrow \infty, x_{2}=0,
$$

then 


$$
|e(x, h)| \leqq K h^{\nu}, \quad \nu<1 / 2, \nu \leqq \eta .
$$

10. Concluding Remarks. In the case when $\sigma \in \partial R_{2}$ is such that

$$
\min _{x \in \partial R_{1}}|\sigma-x| \geqq \delta_{0} \quad \text { (a positive constant) }
$$

one expects the results to be as good as for the Dirichlet problem. We discuss an approach here that deals with case $n=2$ adequately. Define a function

$$
\psi(x)=\gamma \rho(x)^{\beta} \quad \text { with } \rho^{2}=|x|^{2}+\alpha h^{2}
$$

where $\alpha$ and $\gamma$ are constants satisfying certain requirements given below, $0<\beta<1$, and $\sigma$ is again the origin. For $x \in R_{h}{ }^{\prime}$ it is easily verified that

$$
-\Delta_{h} \psi(x) \leqq-|x|^{\beta-2}, \quad x \in R_{h}{ }^{\prime},
$$

provided we choose $\alpha, \gamma$ such that for $c^{2}<1$,

$$
\begin{gathered}
\rho(x)^{2} \geqq c^{2} \rho(\zeta)^{2}, \quad x \in R_{h}, \zeta \in L(x), \\
\alpha>c^{\beta-4} / 2
\end{gathered}
$$

and

$$
\gamma\left(1+\alpha / a^{2}\right)^{(\beta-2) / 2}>1 .
$$

We have a similar inequality for $x \in R_{h}{ }^{*}$ if $\alpha$ further satisfies

$$
\alpha>\left(\frac{4(2-\beta) c^{\beta-3}}{\beta}\right)^{2} .
$$

Putting these together in the representation formula we will arrive at

$$
\begin{aligned}
& h^{2} \sum_{y \in R h^{*}} R(x, y, h)|y-\sigma|^{-2} \leqq K \sum_{y \in \partial R_{1}} R(x, y, h)|y-\sigma|^{\beta-1}+K, \\
& h^{2} \sum_{y \in R_{h}} R(x, y, h)|y-\sigma|^{\beta-2} \leqq K \sum_{y \in \partial R_{1}} R(x, y, h)|y-\sigma|^{\beta-1}+K .
\end{aligned}
$$

Hence if (10.1) is satisfied, then taking $M=3$ and operators $\Delta_{h}{ }^{(1)}$ and $\delta_{1},(10.8)$ yields

$$
|e(x, h)| \leqq K\left(\delta_{0}, \epsilon\right)\left[h^{m+\lambda-\epsilon}+h\right] .
$$

In conclusion we wish to point out that there are several special cases where even when $\sigma \in \partial R_{1}$, convergence results are possible. For instance, if $\partial R_{1}$ includes a rectilinear part and if $\sigma$ happens to be on this part then by placing the grid appropriately and taking simple first-order approximations to the normal derivative on the rectilinear part, we can obtain bounds exactly as in (10.9). This is so because the Robin's function can be bounded by an essentially logarithmic function. Also when rectilinear arcs meet at corners with interior angles not exceeding $\pi / 2$, similar bounds are obtained by adding further logarithmic functions with poles strategically placed outside the region. These little findings encourage us to conjecture that bounds for $R(x, y)$ exist which are less singular than $K|x-y|^{-n+1}$ for general boundaries. This would mean when $n=2$, for example, that even if the solution is only Hölder continuous at some points of $\partial R_{1}$, convergence can be achieved for certain values of $\lambda$ 
Bellcomm, Inc.

Washington, D. C. 20024

1. E. Batschelet, "Über die numerische Auflösung von Randwertproblemen bei elliptischen partiellen Differentialgleichungen," Z. Angew. Math. Phys., v. 3, 1952, pp. 165-193. MR 15, 747.

2. J. H. BRAMBLE \& B. E. HubBaRD, "Approximation of solutions of mixed boundary value problems for Poisson's equation by finite differences," J. Assoc. Comp. Mach., v. 12, 1965, pp. 114-123. MR 30 \#1615.

3. J. H. Bramble \& B. E. Hubbard, "New monotone type approximations for elliptic problems," Math. Comp., v. 18, 1964, pp. 349-367. MR 29 \#2982.

4. J. H. BRAMBLE \& B. E. HUBBARD, "Discretization error in the classical Dirichlet problem for Laplace's equation by finite difference methods," Technical Note BN-484, Institute for Fluid Dynamics and Applied Mathematics, University of Maryland, 1967.

5. J. H. Bramble, B. E. Hubbard \& M. Zlamal, "Discrete analogs of the Dirichlet problem with isolated singularities," SIAM J. Numer. Anal., v. 5, 1968, pp. 1-25.

6. A. Friedman, Partial Differential Equations of Parabolic Type, Prentice-Hall, Englewood Cliffs, N. J., 1964. MR $31 \# 6062$.

7. D. Greenspan, "On the numerical solution of problems allowing mixed boundary conditions," J. Franklin Inst., v. 277, 1964, pp. 11-30. MR 28 \#5569.

8. B. E. Hubbard, "Remarks on the order of convergence in the discrete Dirichlet problem," Proc. Sympos. Numerical Solution of Partial Differential Equations, (Univ. of Maryland, 1965), Academic Press, New York, 1966. MR 35 \#2500.

9. P. LAASONEN, "On the behavior of the solution of the Dirichlet problem at analytic corners," Ann. Acad. Sci. Fenn. Ser. A.I., no. 241, 1957, pp. 1-13. MR 19, 964.

10. P. LaAsonen, "On the solution of Poisson's difference equations," J. Assoc. Comput. Mach., v. 5, 1958, pp. 370-382. MR 22 \#12726. 1966.

11. I. N. SnedDon, Mixed Boundary Value Problems of Potential Theory, Wiley, New York,

12. R. V. Viswanathan, "Solution of Poisson's equation by relaxation method-normal gradient specified on curved boundaries," Math. Comp., v. 11, 1957, pp. 67-78. MR 19, 178.

13. E. A. Volkov, "On the method of nets for a boundary problem with an oblique and a normal derivative," Ż. Vyčisl. Mat. $i$ Mat. Fiz., v. 2, 1962 = U.S.S.R. Comput. Math. and Math. Phys., v. 2, 1962, p. 705.

14. J. A. Voytuk \& R. C. MacCamay, "Mixed boundary-value problems in the plane," Proc. Amer. Math. Soc., v. 16, 1965, pp. 276-280. MR $30 \# 3315$.

15. J. L. WALSH \& D. YounG, "On the degree of convergence of solutions of difference equations to the solution of the Dirichlet problem," J. Mathematical Phys., v. 33, 1954, pp. 80-93. MR $15,746$.

16. W. WAsow, "The accuracy of difference approximations to plane Dirichlet problem with piecewise analytic boundary values," Quart. Appl. Math., v. 15, 1957, pp. 53-63. MR 19, 582.

17. N. M. WigLEY, "Asymptotic expansions at a corner of solutions of mixed boundary value problems," J. Math. Mech., v. 13, 1964, pp. 549-576. MR 29 \#2516.

18. N. M. WIGLEY, "On the convergence of discrete approximations to solutions of mixed boundary value problems," SIAM J. Numer. Anal., v. 3, 1966, pp. 372-382. MR 34 \#5327. 